\title{
Testing natural language use: insights from naturalistic experimental paradigms
}

Katerina Danae Kandylaki

Department of Germanic Linguistics, University of Marburg, Germany

https://doi.org/10.36505/ExLing-2015/06/0006/000243

\begin{abstract}
Experimental approaches to language processing should pay special attention to natural language use. Traditional experimental paradigms use controlled, carefully created stimuli, simplified by decontextualisation. Real language use, however, is full of elliptical, ambiguous sentences where meaning inference rests on contextual cues, and requires not only structural and semantic interpretation for memory storage, but is used to inform actions and interactions. Cognitive neuroscience research is slowly starting to include natural language use in experimental paradigms, by using so called naturalistic paradigms. The current paper presents a naturalistic approach to test language processing and compares results from the current approach to traditional approaches. In conclusion, insights from naturalistic paradigms can inform linguistic theory on the basis of human brain processes.
\end{abstract}

Key words: naturalistic paradigms, online language processing, fMRI

\section{Two traditions of experimental linguistics}

Inspired by experimental sciences and by theoretical and descriptive linguistics, psycho- and neurolinguists often formulate hypotheses that are based on theoretical categorisations of linguistic systems. This, however, might not mirror the way in which our brain categorises language.

The "controlled stimuli" tradition follows strict design principles from psychological science. Researchers create or select carefully controlled stimuli that usually range from single words to maximally two-sentence passages. Then they manipulate certain aspects of the stimuli, according to their hypotheses e.g. the 'b' vs. 'd' distinction in phonology, active vs. passive voice in syntax and test the hypotheses by calculating differential contrasts. In these designs, the participants are usually asked to deliver some judgement (e.g. for the 'b' vs. 'd' distinction, they might be asked to state if the presented word is a pseudoword) or respond to a probe (e.g. as in questions "Was this word in the list you encountered previously?"). Nuisance factors are eliminated as far as possible from the design by the careful control of presentation (randomisation of trials) and by the existence of enough fillers. Traditional statistical approaches (t-tests, chi-squared tests) are applied to analyse the results. In the end, if the experiment was diligently constructed and carried out, the conclusions can be drawn with respect to the initial hypotheses. It is, however, an open question if such findings can scale up to allow conclusion about real language use.

ExLing 2015: Proceedings of 6th Tutorial and Research Workshop on Experimental Linguistics, 26-27 June 2015, Athens, Greece 
The necessity of the alternative approach of the "ecological validity" tradition is underlined by the inevitable abstraction of controlled experimental paradigms, which create an experimental reality, far removed from our everyday linguistic reality. This tradition has been boosted increasingly in the last 15 years by new methodological advances in cognitive neuroscience (e.g. Bartels \& Zeki, 2004; Hasson, Nir, Fuhrmann, \& Malach, 2004) which allow us to present more complex stimuli. In the "naturalistic" paradigms (Willems, 2015), participants are presented with stimuli that capture natural language use as in reading (e.g. Kurby \& Zacks, 2012, 2013), listening to stories (e.g. AbdulSabur et al., 2014), or even communicating in a dialogue (e.g. Kuhlen, Allefeld \& Haynes, 2012). These approaches are characterised by greater freedom in participants' behavior and loss of strict experimental control. In one example experimental situation, a pair of participants is given a problem and they have to interact in order to solve it. What is measured can be the common lexical elements that the two participants use and whether the number of these elements increases as progress is made towards the solution in order to achieve a more efficient collaboration (alignment in dialogue). At the very end of the naturalistic approaches continuum lie completely data-driven experiments as in Bartels \& Zeki (2004). In this study, the results are not modeled by an a priori predictor model, but are derived automatically from the fMRI data by using an algorithm that identifies spatial and temporal activation dynamics during "free-viewing" of a movie.

Although these two traditions might initially seem impossible to combine, recent attempts have been made to combine ecologically valid designs in which controlled stimuli are "hidden" (see for example Haupt, Schlesewsky, Roehm, Friederici, \& Bornkessel-Schlesewsky, 2008). In this way, we take advantage of a controlled experimental design and can still draw conclusions that have a higher probability of applying to natural language use.

\section{The current approach}

In more detail, the current approach uses a story listening paradigm in which participants listen to 20 stories in a functional magnetic resonance imaging (fMRI) experiment. The stories provide a rich auditory language stream of information which allows for a novel manipulation of several linguistic factors in the same natural context. After each story, its content was tested in two comprehension questions. The participants' task was to listen to the stories carefully and answer the two subsequent questions after each story. For the purposes of the current paper we would like to elaborate mainly on the stimuli and one part of the analyses, in order to highlight the advantages of a combined naturalistic approach.

The stories include the following linguistic manipulations: 1. phonological rhythm processing, 2. syntactic and semantic cues for reference tracking and 3. discourse processing of false-belief passages. We managed to embed highly 
controlled experimental conditions in this naturalistic setting by including the manipulations of our factors and filling the rest of the stories context that was as natural as possible. Each of our factors constrained the stimuli at a different level 1. specific compound words for the manipulation of rhythm processing, 2. specific transitive verbs for the reference tracking question, 3. specific discourse for the false belief situations, where one person in the story needed to have a false belief (Frith \& Frith, 1999).

When analysing the data we aimed at describing as many processes as possible, in order to achieve a clean baseline. Therefore, similarly to a mixed effects modelling approach in which random factors are added in order to regress out as much noise as possible, we modelled: 1 . the speech within the stories which was irrelevant to each manipulation, 2. question reading, 3. the response and 4. inter- and intra-trial jitters.

In the current paper we report results on the rhythmic processing manipulation as an example of testing natural language use. Domahs, Klein, Huber, \& Domahs (2013) have conducted a controlled study with similar research questions and found activation for rhythmic processing in the following brain regions: bilateral superior temporal gyrus (STG), right precuneus, left angular gyrus, bilateral inferior frontal gyrus (IFG), bilateral supplementary motor area (SMA), left insula. We found comparable brain regions in a similar manipulation: bilateral STG, right IFG, bilateral SMA and left insula. Moreover, we found additional brain regions which support rhythmic processing in language in context: left premotor cortex (PMC), right post-central gyrus. In the Domahs et al. (2013) study participants were instructed "to decide whether the auditory probe was stressed correctly and as expected given the visual target". In our study participants only listened to the stories without specifically being instructed to pay attention to the stress pattern of the stimuli. Our results therefore establish the ecological validity of the Domahs et al. (2013) study and add to the previously identified rhythm processing regions the PMC and postcentral gyrus.

\section{Conclusion}

Naturalistic designs are necessary for the enrichment of experimental linguistic research, if our goal is to understand language performance. The way in which the brain processes and analyses linguistic input might not be captured in our current theories of linguistic knowledge which are created by reflecting on linguistic output. The processing of language adds useful insights to the system of linguistic knowledge and can inform applications of linguistic interaction.

\section{Acknowledgements}

I am grateful to my advisor, Prof. Dr. Ina Bornkessel-Schlesewsky, for her close guidance in this project. This research was financed by the ExInit initiative and by the 
LOEWE project "Research focus: exploring fundamental linguistic categories" of University of Marburg.

\section{References}

AbdulSabur, N. Y., Xu, Y., Liu, S., Chow, H. M., Baxter, M., Carson, J., \& Braun, A. R. 2014. Neural correlates and network connectivity underlying narrative production and comprehension: A combined fMRI and PET study. Cortex, 57, 107-127.

Bartels, A., \& Zeki, S. 2004. Functional brain mapping during free viewing of na-tural scenes. Human brain mapping, 21(2), 75-85.

Domahs, U., Klein, E., Huber, W., \& Domahs, F. 2013. Good, bad and ugly word stress-fMRI evidence for foot structure driven processing of prosodic violations. Brain and language, 125(3), 272-282.

Frith, C. D., \& Frith, U. 1999. Interacting minds - a biological basis. Science, 286(5445), 1692-1695.

Hasson, U., Nir, Y., Levy, I., Fuhrmann, G., \& Malach, R. 2004. Intersubject synchronization of cortical activity during natural vision. science, 303(5664), 16341640.

Haupt, F. S., Schlesewsky, M., Roehm, D., Friederici, A. D., \& Bornkessel-Schlesewsky, I. 2008. The status of subject-object reanalyses in the language comprehension architecture. Journal of Memory and Language, 59(1), 54-96.

Kuhlen, A. K., Allefeld, C., \& Haynes, J. D. 2012. Content-specific coordination of listeners' to speakers' EEG during communication. Frontiers in buman neuro-science, 6 .

Kurby, C. A., \& Zacks, J. M. 2012. Starting from scratch and building brick by brick in comprehension. Memory \& cognition, 40(5), 812-826.

Kurby, C. A., \& Zacks, J. M. 2013. The activation of modality-specific representations during discourse processing. Brain and language, 126(3), 338-349.

Willems, R. M. (Ed.). 2015. Cognitive neuroscience of natural language use. Cambridge University Press. 